\title{
Influence of Spacing and Planting System on Light Interception, Physiological Parameters, Yield and Quality of Litchi cv. Shahi
}

\author{
Jyoti Singh $^{1 *}$, S. K. Pandey ${ }^{1}$, H. K. Rai ${ }^{1}$, Vishal Nath ${ }^{2}$, \\ Evening Stone Marboh $^{2}$ and Shiv Poojan ${ }^{3}$ \\ ${ }^{1}$ Department of Horticulture, JNKVV Jabalpur (MP), India \\ ${ }^{2}$ NRC on Litchi, Muzaffarpur (Bihar), India \\ ${ }^{3}$ ICAR-CISH Lucknow, India \\ *Corresponding author
}

\section{A B S T R A C T}

\begin{tabular}{|l|}
\hline Ke y w o r d s \\
Light interception, \\
Litchi, training \\
systems, Plant \\
spacing, Planting \\
geometry, Hedge \\
row system
\end{tabular}

\section{Introduction}

Litchi (Litchi chinensis Sonn.) an important fruit crop, belongs to family Sapindaceae. This family has about 150 genera and more than 2500 species which are widely grown in tropical and subtropical regions. Litchi is considered as the "queen of the fruits" due to its attractive color, excellent quality, juicy fruit with excellent sugar and acid blend. It is good source of vitamin-C (Lal et al., 2018a) and phenolics (Lal et al., 2018b).
Characteristic pleasant rosy flavor and nutritional value makes litchi fruit exclusively appealing to the consumers. Litchi originated in South China and South Eastern Asia. India is the second largest producer of litchi in the world after China with an area and production of 1,00,000 ha and 700000 tonnes, respectively (Anonymous, 2019). In India, Bihar, West Bengal, Jharkhand and Assam accounts for $64.2 \%$ of the total litchi production in the country, (Anonymous, 2015). 
Litchi is highly specific to climatic and soil requirements and probably due to which its cultivation is restricted to the few countries in the world (Kumar et al., 2014). Three types of flowers are produced by the litchi tree, namely functional male flowers $\left(\mathrm{M}_{1}\right)$, which lack ovary and ovules and have six to eight stamens produce much pollen; hermaphrodite flowers or functional female (F), that functions as female with a well developed pistil and stigma and five to eight stamens located below the ovary making the condition of heterostyled. The cultivars of litchi does not follow regular pattern of flowering during young stages (Lal et al., 2019d). However, fluctuation in temperature significantly affects fruit retention in litchi (Lal et al., 2017). Fruit load affects the quality production (Nagraj et al., 2019). Thus, High Density Planting is being used to reduce crop load in a single plant and increases production by accommodation of maximum number of plants per unit area. The success of high density planting in litchi crop is due to proper management of orchard in general and litchi in particular. High density planting system depends on maintaining a balance between tree architectural designs, vegetative growth and fruiting vigor of plant. If the plant is less vigorous then excessive fruiting occurs therefore, fruit size decline and incidence of biennial bearing increases and trees fail to fill their allotted space soon enough to make the orchard profitable. If the vegetative vigor is excessive then flowering and fruiting are reduced and containment of the tree to the allotted space becomes problematic (Pandey et al., 2015). To increase litchi production and optimize fruit quality, it is very important to choose the correct training system and optimum plant spacing to obtain good light interception and photosynthetic radiation (Hampson et al., 2002). This combination tends to increase profitability by improving yield (Robinson, 2008). High density planting and training systems in subtropical fruit trees has been an important development in recent years leading to increased productivity, higher early yields and better income per unit area. The possibilities of high density planting systems in fruit crops have been studied by Das et al., (2012), Johnson, et al., (2000), Singh, et al., (2007), Lal et al., (2014), Bal et al., (2003), Kumawat et al., (2014) Balasubramanyan et al., (1997) and Kumari et al., (2003). Under high density planting system, light and microclimatic conditions are important aspects which directly or indirectly affect the vegetative growth, yield and quality of guava fruits. (Brar et al.,2009). Under high density planting system, light is an important aspect which affects the vegetative growth, yield and fruit quality. Novel architectures that enhance light interception and distribution into the canopy have been developed, ensuring early cropping, high yield, improved cropping efficiency and fruit quality (Lauri and Claverie, 2005; Long et al., 2005; Whiting, 2006). Light interception was more in litchi plants planted at wider spacing and decrease significantly with the depth of the canopies irrespective of the planting densities. It has been reported that fruit yield and quality of litchi fruits decreased with poor light interception at higher planting densities. Different training system and planting spacing plays an important role in light interception and its distribution within the tree canopy. Light interception plays crucial role in fruit tree productivity. It influences the flower initiation process and has an impact on fruit quality. Maximum potential assimilation is realized by a canopy that absorbs all incoming light (Wagenmakers, 1995). Fruit yield per ground unit area is positively correlated with light interception. Insufficient light interception and exposition may reduce fruit size, color and total soluble solids (Robinson $e t$ al., 1991; Palmer et al., 1992). Therefore, the present investigations were conducted to study the interception of light under various training system in high density planting in litchi. 


\section{Materials and Methods}

The present investigations were carried out at the ICAR- National Research Centre on litchi, Muzaffarpur (Bihar) during 2018 to 2019 on twelve to fifteen year old litchi plants cv. Shahi. Litchi plants of cv. Shahi planted under different spacing in square and rectangular system (Table 1) with two sets of experiments. The experimental field was sandy loam in texture, alkaline in reaction with low to medium in fertility status with high organic matters with balanced ratio of nitrogen, phosphorous, potash and carbon. The experiment was laid out in Completely Randomized Block Design with 6 treatment combinations replicated four times in both square and rectangular system of planting in litchi. The planting spacing at $8 \times 8 \mathrm{~m}$ was used as control for both the planting systems. These spacing is common and conventional system so comparisons with new planting densities become effective and informative for present experiment. The experimental observations recorded are light interception below and above canopy, canopy area, leaf area $\left(\mathrm{cm}^{2}\right)$, leaf temperature, photosynthetic rate, number of fruits per plant, fruit yield $(\mathrm{kg} / \mathrm{plant})$, fruit yield $(\mathrm{t} / \mathrm{h})$ and fruit quality, fruit weight, fruit length and breadth. The data was subjected to analysis of variance as suggested by Panse and Sukhatme (1967). Significance was tested by ' $F$ ' value at 5 per cent level of probability. Critical difference (CD) values were calculated wherever the $\mathrm{F}$ test was found to be significant.

\section{Treatments details}

The observation on light interception were recorded three times a day at 2 to $3 \mathrm{~h}$ before noon, at noon and 2 to $3 \mathrm{~h}$ after solar noon on completely cloudy, overcast days and on sunny, clear days. Quantum sensor was used to record the light interception. To estimate the light interception the observations were recorded from three spots centre (near the trunk), mid canopy and periphery. The reference of absolute light intensity was measured from the open area at the same time of respective observation. Light interception per tree was estimated by calculating for each below canopy reading the percentage of the above - canopy readings (i.e., transmission), and then by subtracting the average percentage transmission of all 90 sensor reading from $100 \%$ (total incident light).

Formula,

\section{Light Interception $\%=\mathrm{La}-\mathrm{Lb}$}

Where, La is Light available in open area (absolute transmission)

Lb is Light available below the canopy.

Twenty fully expanded leaves were collected at random from each tree and leaf area was measured with the help of Leaf Area Meter (CI-203 Area Meter) and expressed in square centimeter $\left(\mathrm{cm}^{2}\right)$. Photosynthetic rate and leaf temperature was observed by portable photosynthetic system (CIRAS-3) during March - April between 8-9 am. Total numbers of fruits from each experimental tree were counted in the month of May and the average was worked out. Fruit yield $(\mathrm{kg})$ per plant was computed by multiplying the total number of fruits harvested from each plant with the mean fruit weight at the time of harvest and their means were presented during both the years. Fruit length and width of 10 sample fruits from each treatment were measured with the help of digital vernier callipers and expressed in $\mathrm{mm}$. Weight of above sampled fruit was taken on physical balance and average was expressed as gram per fruit. Volume of fruit was measured with the help of volumetric flask and expressed in $\mathrm{ml}$. The fruit weight of each treatment was recorded and total fruits yield per ha was calculated at the final harvesting and 
expressed as t/ha. Bio-chemical parameters viz; total soluble solids were estimated at ambient temperature by digital hand refractometer. Fruit acidity was calculated by titrating the fruit pulp extract with $0.1 \mathrm{~N}$ $\mathrm{NaOH}$ using phenolphthalein indicator (Ranganna, 2010). The data were subjected to statistical analysis as per the method of Gomez and Gomez (1984). Least significant of difference at $5 \%$ level was used for finding the significance of differences among the treatment means.

\section{Results and Discussion}

Data presented in table 1 and table 2 reveals that light interception (\%) in the different part of the canopy (below and upper canopy) significantly influenced by spacing and planting system. Significant increase in light interception (upper and below) was recorded with increase in plant spacing from $2 \times 2 \mathrm{~m}$ (47.8\% below canopy, $84.52 \%$ upper canopy) to $6 \times 6 \mathrm{~m}(74.97 \%$ below $94.02 \%$ above $)$ and control $(80.22 \%$ below and $96.88 \%$ upper). Singh (2001) reported that in Peach cv. Shani- Punjab the maximum radiation interception was found in trees spaced at $6 \times 6 \mathrm{~m}$ than the trees spaced at $3 \times 3 \mathrm{~m}$, both trained to the modified leader system. The upper part of canopy intercepted significantly higher mean light compared to below part of canopy. In general, the lower part of the tree canopy intercepted the least light in all the planting distances. The similar findings were also obtained in the study by Heinicke (1963) and Looney (1968) who also found rapid decrease in light intensity with increasing depth of foliage and very low light intensities at the lower position and central portions of the tree canopy. Jackson (1970) also found more rapid decline in light levels with the depth of tree canopy in apple. Data presented in table 2 shows the maximum light interception recorded in $8 \times 8 \mathrm{~m}(80.22 \%$ upper canopy, $96.88 \%$ below) than $8 \times 4 \mathrm{~m}$ under hedge row system (68.2\% below, 92.25\% upper). Rectangular planting at $4 \times 3 \mathrm{~m}$ with supporting system show higher percentage of light interception $(59.8 \%$ below, $85.02 \%$ upper) than hedge row system at $5 \times 3 \mathrm{~m}$ (54.3\% below, $84.35 \%$ upper) and hedge row system at $6 \times 4 \mathrm{~m}$ (59.4\% below, $87.82 \%$ upper) in the present study.

Table.1 Planting of litchi in square and rectangular system

\begin{tabular}{|c|c|c|c|c|c|}
\hline \multicolumn{3}{|c|}{ Experiment I } & \multicolumn{3}{|c|}{ Experiment II } \\
\hline $\begin{array}{c}\text { Square system of } \\
\text { planting }\end{array}$ & No. of plants/ha & $\begin{array}{l}\text { Space allocation/ } \\
\text { plant }\left(\mathrm{m}^{2}\right)\end{array}$ & $\begin{array}{c}\text { Rectangular } \\
\text { system of planting }\end{array}$ & No. of plants/ha & $\begin{array}{l}\text { Space allocation/ } \\
\text { plant }\left(\mathrm{m}^{2}\right)\end{array}$ \\
\hline $2 \times 2 \mathrm{~m}\left(\mathrm{~T}_{1}\right)$ & 2500 & 4 & $\begin{array}{c}4 \times 3 \mathrm{~m} \text { with } \\
\text { supporting system } \\
\left(\mathrm{T}_{1}\right)\end{array}$ & 834 & 12 \\
\hline $3 \times 3 \mathrm{~m}\left(\mathrm{~T}_{2}\right)$ & 1111 & 9 & $\begin{array}{c}4 \times 3 \mathrm{~m} \text { without } \\
\text { supporting system } \\
\left(\mathrm{T}_{2}\right)\end{array}$ & 834 & 12 \\
\hline $4 \times 4 \mathrm{~m}\left(\mathrm{~T}_{3}\right)$ & 625 & 16 & $\begin{array}{c}5 \times 3 \mathrm{~m} \text { hedge row } \\
\text { system }\left(\mathrm{T}_{3}\right)\end{array}$ & 667 & 15 \\
\hline $5 \times 5 \mathrm{~m}\left(\mathrm{~T}_{4}\right)$ & 400 & 25 & $\begin{array}{l}6 \times 4 \text { m hedge row } \\
\text { system }\left(\mathrm{T}_{4}\right)\end{array}$ & 416 & 24 \\
\hline $6 \times 6 \mathrm{~m}\left(\mathrm{~T}_{5}\right)$ & 278 & 36 & $\begin{array}{l}8 \times 4 \text { m hedge row } \\
\text { system }\left(T_{5}\right)\end{array}$ & 312 & 32 \\
\hline $8 \times 8 m$ (Control) & 156 & 64 & $8 \times 8 \mathrm{~m}$ (control) & 156 & 64 \\
\hline
\end{tabular}


Table.2 Effect of square system of planting on light interception (\%) in litchi

\begin{tabular}{|c|c|c|}
\hline Treatments & $\begin{array}{c}\text { Light interception \% below } \\
\text { canopy }\end{array}$ & $\begin{array}{c}\text { Light interception \% } \\
\text { upper canopy }\end{array}$ \\
\hline & Mean & Mean \\
\hline $\mathbf{2 x 2 ~ m}$ & 47.80 & 84.52 \\
\hline $\mathbf{3 x 3 m}$ & 57.92 & 88.25 \\
\hline $\mathbf{4 x 4 m}$ & 72.97 & 90.05 \\
\hline $\mathbf{5 x 5 m}$ & 75.82 & 93.62 \\
\hline $\mathbf{6 x 6 m}$ & 74.97 & 94.02 \\
\hline $\mathbf{8 x 8 m}$ & 80.22 & 96.88 \\
\hline C.D. at 5\% & 4.748 & 3.631 \\
\hline SE(m) & 1.561 & 1.194 \\
\hline
\end{tabular}

Table.3 Effect of rectangular system of planting on light interception (\%) in litchi

\begin{tabular}{|c|c|c|}
\hline Treatments & $\begin{array}{c}\text { Light interception\% } \\
\text { below canopy }\end{array}$ & $\begin{array}{c}\text { Light interception } \\
\text { upper canopy }\end{array}$ \\
\hline & Mean & Mean \\
\hline 4x3m with support & 59.80 & 85.02 \\
\hline $\mathbf{4 x 3 m}$ without support & 58.92 & 87.00 \\
\hline $\mathbf{5 x 3 m}$ & 54.32 & 84.35 \\
\hline $\mathbf{6 x 4 m}$ & 59.40 & 87.82 \\
\hline $\mathbf{8 x 4 m}$ & 68.20 & 92.22 \\
\hline $\mathbf{8 x 8 m}$ & 80.22 & 96.88 \\
\hline C.D.at 5\% & 2.84 & 4.968 \\
\hline SE(m) & 0.934 & 1.633 \\
\hline
\end{tabular}

Table.3 Effect of square system of planting on physiological, yield and quality traits of litchi

\begin{tabular}{|c|c|c|c|c|c|c|c|c|}
\hline Treatments & $\begin{array}{l}\text { Leaf } \\
\text { area(c } \\
\text { m2) }\end{array}$ & $\begin{array}{c}\text { Photosyntheti } \\
\text { c rate }(\mu \\
\left.\mathrm{mol} / \mathrm{m}^{2} / \mathrm{sec}\right)\end{array}$ & $\begin{array}{c}\text { Leaf } \\
\text { temperat } \\
\text { ure }\left({ }^{0} \mathrm{C}\right)\end{array}$ & $\begin{array}{c}\text { Total } \\
\text { number of } \\
\text { fruits per } \\
\text { plant }\end{array}$ & $\begin{array}{l}\text { Fruit Yield } \\
\text { (kg/plant) }\end{array}$ & $\begin{array}{l}\text { Fruit } \\
\text { yield } \\
\text { (t/h) }\end{array}$ & $\begin{array}{l}\text { Acidi } \\
\text { ty of } \\
\text { fruit } \\
(\%)\end{array}$ & $\begin{array}{c}\text { TSS of } \\
\text { fruit }\left({ }^{0} \mathrm{Brix}\right)\end{array}$ \\
\hline & Mean & Mean & Mean & Mean & Mean & Mean & Mean & Mean \\
\hline $2 \times 2 m$ & 35.32 & 1.70 & 27.86 & 230.62 & 3.65 & 8.26 & 0.91 & 18.15 \\
\hline $3 \times 3 m$ & 35.58 & 2.17 & 29.10 & 470.80 & 6.10 & 7.10 & 0.72 & 19.15 \\
\hline $4 \times 4 m$ & 35.53 & 3.87 & 30.20 & 791.67 & 14.05 & 8.56 & 0.73 & 19.00 \\
\hline $5 \times 5 m$ & 35.58 & 4.92 & 31.13 & $1,146.29$ & 20.97 & 8.29 & 0.80 & 20.19 \\
\hline $6 \times 6 m$ & 35.63 & 6.35 & 31.57 & $2,377.90$ & 40.12 & 10.13 & 0.81 & 20.73 \\
\hline 8x8m & 35.10 & 8.32 & 38.82 & $4,201.14$ & 50.05 & 8.216 & 0.73 & 20.19 \\
\hline C.D. at 5\% & NS & 0.898 & 1.037 & 415.695 & 1 & 0.575 & 0.09 & 1.207 \\
\hline SE(m) & 0.179 & 0.295 & 0.341 & 136.66 & 0.329 & 0.189 & 0.03 & 0.397 \\
\hline
\end{tabular}


Table.4 Effect of rectangular system of planting on physiological, yield and quality traits of litchi

\begin{tabular}{|c|c|c|c|c|c|c|c|c|}
\hline Treatments & $\begin{array}{l}\text { Leaf Leaf } \\
\text { area }\left(\mathrm{cm}^{2}\right)\end{array}$ & $\begin{array}{c}\text { Photosynthetic } \\
\text { rate }(\mu \\
\left.\mathrm{mol} / \mathrm{m}^{2} / \mathrm{sec}\right)\end{array}$ & $\begin{array}{c}\text { Leaf } \\
\text { temperat } \\
\text { ure }\left({ }^{0} \mathrm{C}\right)\end{array}$ & $\begin{array}{c}\text { Total } \\
\text { number of } \\
\text { fruits per } \\
\text { plant }\end{array}$ & $\begin{array}{l}\text { Fruit Yield } \\
\text { (kg/plant) }\end{array}$ & $\begin{array}{l}\text { Fruit } \\
\text { yield } \\
(\mathbf{t} / \mathbf{h})\end{array}$ & $\begin{array}{c}\text { Acidity } \\
\text { of fruit } \\
(\%)\end{array}$ & $\begin{array}{l}\text { TSS of } \\
\text { fruit }{ }^{0} \\
\text { Brix) }\end{array}$ \\
\hline & Mean & Mean & Mean & Mean & Mean & Mean & Mean & Mean \\
\hline $\begin{array}{c}4 \times 3 \text { m with } \\
\text { support }\end{array}$ & 35.79 & 4.82 & 29.42 & 735.63 & 16.51 & 12.88 & 0.79 & 19.56 \\
\hline $\begin{array}{l}4 \times 3 \text { m without } \\
\text { support }\end{array}$ & 36.17 & 4.55 & 29.65 & 455.70 & 8.86 & 11.67 & 0.77 & 19.08 \\
\hline $5 \times 3 \mathrm{~m}$ & 35.60 & 5.12 & 29.13 & 546.50 & 11.22 & 7.48 & 0.85 & 19.41 \\
\hline $6 \times 4 \mathrm{~m}$ & 35.22 & 5.70 & 30.01 & 3002.43 & 33.38 & 15.87 & 0.77 & 20.46 \\
\hline $8 \times 4 m$ & 36.35 & 6.90 & 35.97 & 3561.87 & 42.35 & 20.33 & 0.64 & 21.17 \\
\hline $8 x 8$ m & 35.10 & 8.32 & 38.82 & 4201.14 & 50.05 & 8.22 & 0.74 & 20.19 \\
\hline C.D. at $5 \%$ & NS & 0.983 & 1.791 & 408.257 & 3.067 & 3.827 & 0.078 & 0.761 \\
\hline SE(m) & 0.379 & 0.323 & 0.589 & 134.215 & 1.008 & 1.258 & 0.026 & 0.25 \\
\hline
\end{tabular}

Table.5 Effect of square system on canopy area and yield attributes of litchi

\begin{tabular}{|c|c|c|c|c|}
\hline Treatments & $\begin{array}{l}\text { Mean fruit } \\
\text { weight (g) }\end{array}$ & $\begin{array}{l}\text { Mean fruit length } \\
(\mathbf{m m})\end{array}$ & $\begin{array}{l}\text { Mean fruit } \\
\text { breath(mm) }\end{array}$ & $\begin{array}{c}\text { Canopy } \\
\operatorname{area}\left(\mathbf{m}^{2}\right)\end{array}$ \\
\hline $2 \times 2 m$ & 19.173 & 32.68 & 29.21 & 6.85 \\
\hline $3 \times 3 m$ & 20.065 & 33.34 & 27.71 & 4.99 \\
\hline $4 \times 4 m$ & 20.608 & 34.015 & 28.64 & 11.67 \\
\hline $5 \times 5 m$ & 21.208 & 34.20 & 29.46 & 14.98 \\
\hline $6 \times 6 \mathrm{~m}$ & 22.733 & 36.74 & 31.05 & 24.79 \\
\hline $8 \times 8 m$ & 21.778 & 35.26 & 28.35 & 31.74 \\
\hline C.D. at $5 \%$ & 1.246 & 1.152 & 1.312 & 2.848 \\
\hline SE(m) & 0.41 & 0.379 & 0.431 & 0.936 \\
\hline
\end{tabular}

Table.6 Effect of rectangular system on canopy area and yield attributes of litchi

\begin{tabular}{|c|c|c|c|c|}
\hline Treatments & $\begin{array}{c}\text { Mean fruit } \\
\text { weight (g) }\end{array}$ & $\begin{array}{l}\text { Mean fruit } \\
\text { length }(\mathrm{mm})\end{array}$ & $\begin{array}{c}\text { Mean fruit } \\
\text { breath }(\mathbf{m m})\end{array}$ & $\begin{array}{r}\text { Canopy } \\
\operatorname{area}\left(\mathbf{m}^{2}\right)\end{array}$ \\
\hline $4 \times 3 \mathrm{~m}$ with support & 19.658 & 33.054 & 26.573 & 10.953 \\
\hline $4 \times 3$ m without support & 19.856 & 32.944 & 26.883 & 7.038 \\
\hline $5 \times 3 \mathrm{~m}$ & 20.723 & 35.075 & 29.02 & 10.769 \\
\hline $6 \times 4 \mathrm{~m}$ & 22.329 & 36.514 & 31.545 & 15.75 \\
\hline $8 \times 4 \mathrm{~m}$ & 23.485 & 40.075 & 33.05 & 28.638 \\
\hline $8 \times 8 \mathrm{~m}$ & 21.776 & 35.259 & 28.749 & 31.744 \\
\hline C.D.at 5\% & 0.933 & 0.962 & 1.53 & 2.986 \\
\hline SE(m) & 0.307 & 0.301 & 0.503 & 0.98 \\
\hline
\end{tabular}


Pearson Correlation Matrix (Effect of Square system on Litchi)

\begin{tabular}{|c|c|c|c|c|c|c|c|c|c|c|c|c|c|}
\hline & $\begin{array}{l}\text { Leaf } \\
\text { area } \\
(\mathrm{cm} 2)\end{array}$ & $\begin{array}{c}\text { number of } \\
\text { fruit per } \\
\text { plant }\end{array}$ & $\begin{array}{l}\text { Fruit } \\
\text { Yield } \\
\text { (kg/ } \\
\text { plant) }\end{array}$ & $\begin{array}{c}\text { Fruit } \\
\text { yield }(t / h)\end{array}$ & $\begin{array}{l}\text { Acidity } \\
\text { of fruit }\end{array}$ & $\begin{array}{l}\text { TSS of } \\
\text { fruit }\end{array}$ & $\begin{array}{c}\text { LI } \\
\text { below } \\
\text { canopy }\end{array}$ & $\begin{array}{c}\text { LI } \\
\text { upper } \\
\text { canopy }\end{array}$ & $\begin{array}{c}\text { Photosy. } \\
\text { rate }\end{array}$ & $\begin{array}{c}\text { Fruit } \\
\text { weight }\end{array}$ & $\begin{array}{c}\text { Fruit } \\
\text { length }\end{array}$ & $\begin{array}{c}\text { Fruit } \\
\text { breath }\end{array}$ & $\begin{array}{c}\text { Canopy } \\
\text { area }\end{array}$ \\
\hline Leaf area & & $-0.581^{\mathrm{NS}}$ & $-0.389^{\mathrm{NS}}$ & $0.218^{\mathrm{NS}}$ & $-0.039^{\mathrm{NS}}$ & $0.130^{\mathrm{NS}}$ & $-0.053^{\mathrm{NS}}$ & $-0.199^{\mathrm{NS}}$ & $-0.390^{\mathrm{NS}}$ & $0.091^{\mathrm{NS}}$ & $0.076^{\mathrm{NS}}$ & $0.365^{\mathrm{NS}}$ & $-0.454^{\mathrm{NS}}$ \\
\hline $\begin{array}{c}\text { Nu. of } \\
\text { fruit/plant }\end{array}$ & $-0.581^{\mathrm{NS}}$ & & $0.968^{* *}$ & $0.333^{\mathrm{NS}}$ & $-0.273^{\mathrm{NS}}$ & $0.705^{\mathrm{NS}}$ & $0.725^{\mathrm{NS}}$ & $0.866^{*}$ & $0.956^{* *}$ & $0.748^{\mathrm{NS}}$ & $0.733^{\mathrm{NS}}$ & $0.136^{\mathrm{NS}}$ & $0.970^{* *}$ \\
\hline $\begin{array}{l}\text { Fruit yield } \\
\text { (kg) }\end{array}$ & $-0.389^{\mathrm{NS}}$ & $0.968^{* *}$ & & $0.523^{\mathrm{NS}}$ & $-0.216^{\mathrm{NS}}$ & $0.830^{*}$ & $0.804^{\mathrm{NS}}$ & $0.916^{*}$ & $0.984^{* *}$ & $0.882^{*}$ & $0.869^{*}$ & $0.348^{\mathrm{NS}}$ & $0.994^{* *}$ \\
\hline $\begin{array}{c}\text { Fruit yield } \\
(t / h)\end{array}$ & $0.218^{\mathrm{NS}}$ & $0.333^{\mathrm{NS}}$ & $0.523^{\mathrm{NS}}$ & & $0.354^{\mathrm{NS}}$ & $0.525^{\mathrm{NS}}$ & $0.415^{\mathrm{NS}}$ & $0.370^{\mathrm{NS}}$ & $0.465^{\mathrm{NS}}$ & $0.687^{\mathrm{NS}}$ & $0.773^{\mathrm{NS}}$ & $0.922^{* *}$ & $0.533^{\mathrm{NS}}$ \\
\hline Acidity & $-0.039^{\mathrm{NS}}$ & $-0.273^{\mathrm{NS}}$ & $-0.216^{\mathrm{NS}}$ & $0.354^{\mathrm{NS}}$ & & $-0.279^{\mathrm{NS}}$ & $-0.511^{\mathrm{NS}}$ & $-0.430^{\mathrm{NS}}$ & $-0.291^{\mathrm{NS}}$ & $\overline{-}^{-} 254^{\mathrm{NS}}$ & $-0.160^{\mathrm{NS}}$ & $0.567^{\mathrm{NS}}$ & $-0.159^{\mathrm{NS}}$ \\
\hline TSS & $0.130^{\mathrm{NS}}$ & $0.705^{\mathrm{NS}}$ & $0.830^{*}$ & $0.525^{\mathrm{NS}}$ & $-0.279^{\mathrm{NS}}$ & & $0.837^{*}$ & $0.912^{*}$ & $0.831^{*}$ & $0.959^{* *}$ & $0.895^{*}$ & $0.488^{\mathrm{NS}}$ & $0.781^{\mathrm{NS}}$ \\
\hline $\begin{array}{l}\text { LI below } \\
\text { canopy }\end{array}$ & $-0.053^{\mathrm{NS}}$ & $0.725^{\mathrm{NS}}$ & $0.804^{\mathrm{NS}}$ & $0.415^{\mathrm{NS}}$ & $-0.511^{\mathrm{NS}}$ & $0.837^{*}$ & & $0.944^{* *}$ & $0.879^{*}$ & $0.851^{*}$ & $0.755^{\mathrm{NS}}$ & $0.231^{\mathrm{NS}}$ & $0.788^{\mathrm{NS}}$ \\
\hline $\begin{array}{l}\text { LI upper } \\
\text { canopy }\end{array}$ & $-0.199^{\mathrm{NS}}$ & $0.866^{*}$ & $0.916^{*}$ & $0.370^{\mathrm{NS}}$ & $-0.430^{\mathrm{NS}}$ & $0.912^{*}$ & $0.944^{* *}$ & & $0.958^{* *}$ & $0.890^{*}$ & $0.802^{\mathrm{NS}}$ & $0.233^{\mathrm{NS}}$ & $0.894^{*}$ \\
\hline $\begin{array}{c}\text { Photosyn. } \\
\text { Rate }\end{array}$ & $-0.390^{\mathrm{NS}}$ & $0.956^{* *}$ & $0.984^{* *}$ & $0.465^{\mathrm{NS}}$ & $-0.291^{\mathrm{NS}}$ & $0.831^{*}$ & $0.879^{*}$ & $0.958^{* *}$ & & $0.867^{*}$ & $0.825^{*}$ & $0.283^{\mathrm{NS}}$ & $0.983^{* *}$ \\
\hline Frit weight & $0.091^{\mathrm{NS}}$ & $0.748^{\mathrm{NS}}$ & $0.882^{*}$ & $0.687^{\mathrm{NS}}$ & $-0.254^{\mathrm{NS}}$ & $0.959^{* *}$ & $0.851^{*}$ & $0.890^{*}$ & $0.867^{*}$ & & $0.979^{* *}$ & $0.567^{\mathrm{NS}}$ & $0.844^{*}$ \\
\hline Fruit length & $0.076^{\mathrm{NS}}$ & $0.733^{\mathrm{NS}}$ & $0.869^{*}$ & $0.773^{\mathrm{NS}}$ & $-0.160^{\mathrm{NS}}$ & $0.895^{*}$ & $0.755^{\mathrm{NS}}$ & $0.802^{\mathrm{NS}}$ & $0.825^{*}$ & $0.979^{* *}$ & & $0.635^{\mathrm{NS}}$ & $0.835^{*}$ \\
\hline $\begin{array}{c}\text { Fruit } \\
\text { breath }\end{array}$ & $0.365^{\mathrm{NS}}$ & $0.136^{\mathrm{NS}}$ & $0.348^{\mathrm{NS}}$ & $0.922^{* *}$ & $0.567^{\mathrm{NS}}$ & $0.488^{\mathrm{NS}}$ & $0.231^{\mathrm{NS}}$ & $0.233^{\mathrm{NS}}$ & $0.283^{\mathrm{NS}}$ & $0.567^{\mathrm{NS}}$ & $0.635^{\mathrm{NS}}$ & & $0.351^{\mathrm{NS}}$ \\
\hline $\begin{array}{c}\text { Canopy } \\
\text { area }\end{array}$ & $-0.454^{\mathrm{NS}}$ & $0.970^{* *}$ & $0.994^{* *}$ & $0.533^{\mathrm{NS}}$ & $-0.159^{\mathrm{NS}}$ & $0.781^{\mathrm{NS}}$ & $0.788^{\mathrm{NS}}$ & $0.894^{*}$ & $0.983^{* *}$ & $0.844^{*}$ & $0.835^{*}$ & $0.351^{\mathrm{NS}}$ & \\
\hline
\end{tabular}


Pearson Correlation Matrix (Effect of rectangular system on litchi)

\begin{tabular}{|c|c|c|c|c|c|c|c|c|c|c|c|c|c|}
\hline & $\begin{array}{c}\text { Leaf } \\
\operatorname{area}(\mathrm{cm} 2)\end{array}$ & $\begin{array}{c}\text { Total } \\
\text { number } \\
\text { of fruit } \\
\text { per } \\
\text { plant }\end{array}$ & $\begin{array}{c}\text { Fruit } \\
\text { Yield } \\
\text { (kg/plant) }\end{array}$ & $\begin{array}{l}\text { Fruit } \\
\text { yield } \\
(\mathbf{t} / \mathbf{h})\end{array}$ & $\begin{array}{l}\text { Acidity } \\
\text { of fruit }\end{array}$ & $\begin{array}{c}\text { TSS of } \\
\text { fruit }\end{array}$ & $\begin{array}{l}\text { LI below } \\
\text { canopy }\end{array}$ & $\begin{array}{c}\text { LI } \\
\text { upper } \\
\text { canopy }\end{array}$ & Photosy.rate & $\begin{array}{c}\text { Fruit } \\
\text { weight }\end{array}$ & $\begin{array}{c}\text { Fruit } \\
\text { length }\end{array}$ & $\begin{array}{c}\text { Fruit } \\
\text { breath }\end{array}$ & $\begin{array}{c}\text { Canopy } \\
\text { area }\end{array}$ \\
\hline Leaf area & & $0.440^{\mathrm{NS}}$ & $0.467^{\mathrm{NS}}$ & $0.073^{\mathrm{NS}}$ & $-0.053^{\mathrm{NS}}$ & $0.247^{\mathrm{NS}}$ & $0.391^{\mathrm{NS}}$ & $0.291^{\mathrm{NS}}$ & $0.315^{\mathrm{NS}}$ & $0.062^{\mathrm{NS}}$ & $-0.123^{\mathrm{NS}}$ & $-0.053^{\mathrm{NS}}$ & $0.273^{\mathrm{NS}}$ \\
\hline Nu.of fruit/plant & $0.440^{\mathrm{NS}}$ & & $0.992^{* *}$ & $0.346^{\mathrm{NS}}$ & $-0.691^{\mathrm{NS}}$ & $0.846^{*}$ & $0.822^{*}$ & $0.889^{*}$ & $0.918^{* *}$ & $0.840^{*}$ & $0.685^{\mathrm{NS}}$ & $0.668^{\mathrm{NS}}$ & $0.934^{* *}$ \\
\hline Fruityield(kg) & $0.467^{\mathrm{NS}}$ & $0.992^{* *}$ & & $0.319^{\mathrm{NS}}$ & $-0.694^{\mathrm{NS}}$ & $0.834^{*}$ & $0.863^{*}$ & $0.903^{*}$ & $0.941^{* *}$ & $0.801^{\mathrm{NS}}$ & $0.658^{\mathrm{NS}}$ & $0.615^{\mathrm{NS}}$ & $0.960^{* * *}$ \\
\hline Fruit yield (t/h) & $0.073^{\mathrm{NS}}$ & $0.346^{\mathrm{NS}}$ & $0.319^{\mathrm{NS}}$ & & $-0.758^{\mathrm{NS}}$ & $0.721^{\mathrm{NS}}$ & $-0.000^{\mathrm{NS}}$ & $0.097^{\mathrm{NS}}$ & $0.039^{\mathrm{NS}}$ & $0.609^{\mathrm{NS}}$ & $0.685^{\mathrm{NS}}$ & $0.674^{\mathrm{NS}}$ & $0.243^{\mathrm{NS}}$ \\
\hline Acidity & $-0.053^{\mathrm{NS}}$ & $-0.691^{\mathrm{NS}}$ & $-0.694^{\mathrm{NS}}$ & $-0.758^{\mathrm{NS}}$ & & $-0.801^{\mathrm{NS}}$ & $-0.593^{\mathrm{NS}}$ & $-0.670^{\mathrm{NS}}$ & $-0.570^{\mathrm{NS}}$ & $-0.728^{\mathrm{NS}}$ & $-0.735^{\mathrm{NS}}$ & $-0.618^{\mathrm{NS}}$ & $-0.715^{\mathrm{NS}}$ \\
\hline TSS & $0.247^{\mathrm{NS}}$ & $0.846^{*}$ & $0.834^{*}$ & $0.721^{\mathrm{NS}}$ & $-0.801^{\mathrm{NS}}$ & & $0.498^{\mathrm{NS}}$ & $0.594^{\mathrm{NS}}$ & $0.661^{\mathrm{NS}}$ & $0.952^{* *}$ & $0.926^{* *}$ & $0.898^{*}$ & $0.789^{\mathrm{NS}}$ \\
\hline LI below canopy & $0.391^{\mathrm{NS}}$ & $0.822^{*}$ & $0.863^{*}$ & $-0.000^{\mathrm{NS}}$ & $-0.593^{\mathrm{NS}}$ & $0.498^{\mathrm{NS}}$ & & $0.972^{* *}$ & $0.935^{* *}$ & $0.458^{\mathrm{NS}}$ & $0.308^{\mathrm{NS}}$ & $0.192^{\mathrm{NS}}$ & $0.898^{*}$ \\
\hline LI upper canopy & $0.291^{\mathrm{NS}}$ & $0.889^{*}$ & $0.903^{*}$ & $0.097^{\mathrm{NS}}$ & $-0.670^{N S}$ & $0.594^{\mathrm{NS}}$ & $0.972^{* *}$ & & $0.953^{* *}$ & $0.604^{\mathrm{NS}}$ & $0.450^{\mathrm{NS}}$ & $0.360^{\mathrm{NS}}$ & $0.927^{* *}$ \\
\hline Photosyn.rate & $0.315^{\mathrm{NS}}$ & $0.918^{* *}$ & $0.941^{* *}$ & $0.039^{\mathrm{NS}}$ & $-0.570^{\mathrm{NS}}$ & $0.661^{\mathrm{NS}}$ & $0.935^{* *}$ & $0.953^{* *}$ & & $0.674^{\mathrm{NS}}$ & $0.534^{\mathrm{NS}}$ & $0.452^{\mathrm{NS}}$ & $0.973^{* *}$ \\
\hline Frit weight & $0.062^{\mathrm{NS}}$ & $0.840^{*}$ & $0.801^{\mathrm{NS}}$ & $0.609^{\mathrm{NS}}$ & $-0.728^{\mathrm{NS}}$ & $0.952^{* *}$ & $0.458^{\mathrm{NS}}$ & $0.604^{\mathrm{NS}}$ & $0.674^{\mathrm{NS}}$ & & $0.959^{* *}$ & $0.960^{* *}$ & $0.769^{\mathrm{NS}}$ \\
\hline Fruit length & $-0.123^{\mathrm{NS}}$ & $0.685^{\mathrm{NS}}$ & $0.658^{\mathrm{NS}}$ & $0.685^{\mathrm{NS}}$ & $-0.735^{\mathrm{NS}}$ & $0.926^{* *}$ & $0.308^{\mathrm{NS}}$ & $0.450^{\mathrm{NS}}$ & $0.534^{\mathrm{NS}}$ & $0.959^{* *}$ & & $0.965^{* *}$ & $0.675^{\mathrm{NS}}$ \\
\hline Fruit breath & $-0.053^{\mathrm{NS}}$ & $0.668^{\mathrm{NS}}$ & $0.615^{\mathrm{NS}}$ & $0.674^{\mathrm{NS}}$ & $-0.618^{\mathrm{NS}}$ & $0.898^{*}$ & $0.192^{\mathrm{NS}}$ & $0.360^{\mathrm{NS}}$ & $0.452^{\mathrm{NS}}$ & $0.960^{* *}$ & $0.965^{* *}$ & & $0.571^{\mathrm{NS}}$ \\
\hline Canopy area & $0.273^{\mathrm{NS}}$ & $0.934^{* *}$ & $0.960^{* *}$ & $0.243^{\mathrm{NS}}$ & $-0.715^{\mathrm{NS}}$ & $0.789^{\mathrm{NS}}$ & $0.898^{*}$ & $0.927^{\text {** }}$ & $0.973^{* *}$ & $0.769^{\mathrm{NS}}$ & $0.675^{\mathrm{NS}}$ & $0.571^{\mathrm{NS}}$ & \\
\hline
\end{tabular}


It may be due to well distribution of primary, secondary and tertiary branches and opened centre. Singh (2001) and Singh and Dhaliwal (2007), also observed highest radiation interception in the upper canopy part during midday (12.00- $14.00 \mathrm{~h}$ ) hours in peach and guava respectively. The average radiation interception was found to be the maximum in the upper part of the canopy irrespective of spacing and training system and it decreased with the depth of foliage. Heinicke (1963) and Looney (1968) reported in apple that light intensity decreased rapidly with increasing depth of foliage and the lower and central portions of the tree receives very low light intensities. The data on physiological parameters yield and quality traits of litchi at different plant spacing has been presented in table 3 \&4. Data clearly indicates that leaf area $\left(\mathrm{cm}^{2}\right)$, photosynthetic rate, leaf temperature $\left({ }^{0} \mathrm{C}\right)$, total number of fruits per plant, fruit yield $(\mathrm{kg} / \mathrm{h})$ and TSS ( ${ }^{0}$ Brix) increased with increase in planting spacing. Leaf area in both spacing and planting system do not have significant variation (Table $3 \&$ 4). Similar result were also found by Sharma (2015) in peach where both planting systems and spacing had no significant effect on the leaf area of peach trees during her investigations The data reveal that mean leaf area was found to be maximum in $8 \times 8 \mathrm{~m}$ $\left(40.28 \mathrm{~cm}^{2}\right)$ followed by $6 \times 6 \mathrm{~m}\left(40.10 \mathrm{~cm}^{2}\right)$ in square system and $8 \times 4 \mathrm{~m}$ in rectangular system under hedge row $\left(39.53 \mathrm{~cm}^{2}\right)$ of planting system but their difference did not attain a level of significant. The planting system and spacing had no significant effect on the leaf area of litchi trees during the present investigations.

These results are in accordance with reports of Caruso et al., (1999) who also observed that leaf area was not affected by training system in peaches. Significantly maximum number of fruits and yield per plant were found in wider spacing 8x8m (4201.14 and
$50.05 \mathrm{~kg} / \mathrm{plant}$ respectively) followed by $6 \times 6 \mathrm{~m}$ (2377.90 and $40.12 \mathrm{~kg} / \mathrm{plant}$ respectively) among square system and $8 \times 4 \mathrm{~m}$ in hedge row system (3561.87 and $42.35 \mathrm{~kg} /$ plant) over closer spacing. Rectangular system at $8 \times 4 \mathrm{~m}$ and $6 \times 4 \mathrm{~m}$ in hedge row system is significantly superior in terms of yield at $5 \times 5 \mathrm{~m}$ and $6 \times 6 \mathrm{~m}$ covering a space of $25 \mathrm{~m}^{2}$ and $36 \mathrm{~m}^{2}$ which revealed that rectangular system (hedge row system) has better yield potential than square system.

These results have been obtain probably due to higher light interception below and upper part of canopy, moisture and air circulation and availability of nutrients in rectangular system than square system. Sharma (2015) also reported that lower number of fruits per plant at closer plantings may be due to less fruit bearing areas and smaller canopy area. It can also be due to lower flower bud density recorded in closely planted trees during the course of this study. Callesen and Wagenmakers (1989) reported that higher number of fruit per tree at wider spacing was due to higher tree volume and flowering which support the present findings. Various workers (Bellini et al., 2000; Ahmed and Morad 2000; Lu et al., 2003; Rufato et al., 2004) have suggested a close relationship between fruit yield and radiation intercepted in fruit crops. Similarly Mika et al., (2001) observed that trees planted at closer spacing had to compete with each other for light, water and nutrients as a result of which yield decreases. Cepoiu and Muravi (1988) also reported that wider spacing were helpful in increasing yield due to higher tree volume and reduced competition for metabolites among plants. The highest fruit yield per hectare was recorded at $8 \times 4 \mathrm{~m}(20.33 \mathrm{t} / \mathrm{h})$ in hedge row system than $6 \times 6 \mathrm{~m}(10.13 \mathrm{t} / \mathrm{h})$ in square system. Yield in control $(8.21 \mathrm{t} / \mathrm{h})$ was significantly at par with $2 \times 2 \mathrm{~m}, 4 \times 4 \mathrm{~m}$ and $5 \times 5 \mathrm{~m}(8.26,8.56$ and $8.29 \mathrm{t} / \mathrm{h}$ respectively) in square system of planting. Similar studies also 
reported by Sharma et al., (1992) at FRS, Abohar, Punjab, that the effect of plant spacing $(6 \times 3 \mathrm{~m}, 6 \times 4 \mathrm{~m}, 6 \mathrm{x} 6 \mathrm{~m}$ and $7 \times 7 \mathrm{~m})$ in Kinnow mandarin and recorded the highest average fruit yield (141. 9 q/ac) at $6 \times 3 \mathrm{~m}$ spacing. However, a decrease in yield per acre with increase in plant spacing was also observed. Yield per hectare was recorded to be maximum under different hedge row system in rectangular than different square system. This resultsmay be due to maximum number of plants per unit area and maximum interception of light in different rectangular system than square system. These results concur with many studies (Bunea 1982; Bargioni et al., 1986; Costa et al., 1997; Rana et al., 1998) where yield was low with increase in tree density, while the yield per hectare increased. Higher yield per hectare at closer spacing was due to increased number of plants and foliage per hectare. The highest total soluble solids were recorded at $6 \times 6 \mathrm{~m}$ in square system and $8 \times 4 \mathrm{~m}$ in hedge row system in rectangular systems $(20.73 \%$, $21.17 \%$ respectively) followed by $8 \times 8 \mathrm{~m}$ (20.19\%). But rectangular system is more efficient than square system in quality attributes due to maximum light and orientation of plants. Acidity of fruits decreases with increasing in spacing. The lowest acidity $(0.73 \%)$ was recorded in in $6 \times 6$ $\mathrm{m}$ in square system and in $8 \times 4 \mathrm{~m}(0.61 \%)$ in rectangular system closely followed by $8 \times 8 \mathrm{~m}$ (0.68\%). Gaikwad et al., (1981) who studied the relationship of plant density with fruit quality of Sardar guava observed a decrease in total soluble solids and an increase in acid content with increased planting density due to poor interception of light, air circulation and competition for nutrients in dense planting orchard. In physiological data, photosynthetic rate and leaf temperature significantly increases with increasing in spacing. The maximum photosynthetic rate recorded in $8 \times 8$ $\mathrm{m}\left(8.32 \mathrm{umol} / \mathrm{m}^{2} / \mathrm{sec}\right)$ closely followed by $6 \times 6$ $\mathrm{m}\left(6.35 \mathrm{umol} / \mathrm{m}^{2} / \mathrm{sec}\right)$ in square system and
$8 \times 4 \mathrm{~m}$ under hedge row system (6.90 $\mathrm{umol} / \mathrm{m}^{2} / \mathrm{sec}$ ) in rectangular system. It might be due to maximum penetration of light. Leaf temperature also increased with increased spacing. Maximum leaf temperature was observed in $8 \times 8 \mathrm{~m}\left(40.28^{\circ}\right)$ due to more interception of light by below and above part of canopy. It increased with the increase in ambient temperature (Marathe et al., 2017). The present results also show that canopy area in both square and rectangular system increased in wider spaced trees (Table $5 \& 6$ ). The maximum canopy area was observed in $8 \times 8 \mathrm{~m}\left(31.74 \mathrm{~m}^{2}\right)$ closely followed by $6 \times 6 \mathrm{~m}$ $\left(24.79 \mathrm{~m}^{2}\right)$ and $8 \times 4 \mathrm{~m}\left(28.63 \mathrm{~m}^{2}\right)$. Similar finding were also reported by Yadav et al., (1981) in Sardar guava, Boswell (1970) in Washington Naval orange and Arora et al., (1983) in Kinnow where widely spaced trees had more canopy area and volume as compared to closely spaced trees due to increased biomass. The fruit weight, in general, increased with increase in wider spacing. Data revealed in table 5 and 6 that maximum mean fruit weight of $22.73 \mathrm{~g}$ was recorded in fruits harvested from trees spaced at $6 \times 6 \mathrm{~m}$ in square system and $23.48 \mathrm{~g}$ from $8 \times 4 \mathrm{~m}$ under hedge row system in rectangular system and it was significantly more than the fruits harvested from the trees spaced at $8 \times 8$ $\mathrm{m}$ (control).This might be due to more per cent radiation interception on per tree basis in wider spacing and less number of fruits per plant that provides ample space for growth and development of fruit than in $8 \times 8 \mathrm{~m}$ which led to severe competition for metabolites and caused reduction in fruit weight. Maximum mean fruit length and breadth of $36.74 \mathrm{~mm}$, $31.05 \mathrm{~mm}$ respectively was recorded from trees spaced at $6 \times 6 \mathrm{~m}$ in square system in table 5 while in rectangular system the maximum length $(40.07 \mathrm{~mm})$ and breadth $(33.05 \mathrm{~mm})$ was noticed in $8 \times 4 \mathrm{~m}$ under hedge row system and it was significantly higher than the fruits harvested from the trees spaced at $8 \times 8 \mathrm{~m}$ (control) in table 6. Ahmed and Higazi (1983) 
in oranges also observed reduction in fruit size (length and breadth) with increase in tree density whereas, Lal et al., (1997) in Sweet oranges recorded improved fruit size in wider (6x6 m) spacing than in $6 \times 4 \mathrm{~m}$ and $6 \times 5 \mathrm{~m}$ spacing. Reduction in fruit size due to low light exposure subsequently affecting carbohydrates supply is also supported by the findings of Tustin et al., (1989) in apple. Similar result was found by $\mathrm{S}$ Lal et al.,(2018) that lower fruit size in closer spacing in peaches and if agronomical practices such as irrigation or fertilization can be modified to improve fruit size. In square system of planting net photosynthetic rate has highly significant positive correlated with total number of fruits per plant, fruit yield ( $\mathrm{kg} / \mathrm{plant}$ ), and canopy area of plant and significant positive correlation with total soluble solids, weight and length of fruit, negative non significant with leaf area, non significant with fruit acidity, breadth and fruit yield $(\mathrm{t} / \mathrm{ha})$. Light interception upper part of canopy has significant positive correlated with total number of fruits per plant, total soluble solids, fruit weight and canopy area and non significant with leaf area, fruit yield (t/ha), acidity, length and breadth of fruit. Canopy area has highly significant positive correlated with total number of fruits per plant, fruit yield ( $\mathrm{kg} /$ plant) and photosynthetic rate and significant positive correlation with light interception upper canopy, fruit weight, fruit length. Non significant correlated with leaf area, fruit yield (t/ha), acidity, TSS and light interception below canopy. In rectangular system of planting net photosynthetic rate has highly significant positive correlated with number of fruits per plant, fruit yield ( $\mathrm{kg} /$ plant), light interception (upper and below part of canopy) and canopy area and non significant with leaf area, fruit yield (t/ha), TSS, fruit weight, length and breadth, non significant negative correlated with acidity of fruit. Light interception (upper and below canopy) significant positive correlated with number of fruits per plant, fruit yield ( $\mathrm{kg} /$ plant), canopy area, negative correlated with acidity and non significant with leaf area, TSS, fruit weight, length and breadth. Canopy area has highly significant positive correlated with fruit yield (kg/plant), number of fruits per plant and photosynthetic rate.

In conclusion the production of better yield and quality in litchi from less land and efficient harnessing of natural resources is need of the hour where planting geometry and planting system plays a crucial role. The study indicates that planting in rectangular system at spacing of $8 \times 4 \mathrm{~m}$ occupying $32 \mathrm{~m}^{2}$ land space was able to produce an yield of $20.33 \mathrm{t} / \mathrm{ha}$. Therefore, for Shahi litchi, the spacing of $8 \times 4 \mathrm{~m}$ under hedge row system can be recommended for the farmers to obtain higher yield of 19-20 t/ha against 8.21t/ha in normal square system of planting at $8 \times 8 \mathrm{~m}$.

\section{Acknowledgements}

First author is thankful to Prof. and Director Vishal Nath for help in planning and execution of the experiment and providing all the necessary facility for the experiment.

\section{References}

Ahmed M and Morad E: Training system for peach trees [Prunus persica (L.) Batsch] in Morocco. Fruits-Paris 55(2): 153-61. (2000)

Ahmed S A and Higazi AM: Effect of orchard density on yield and fruit quality in Arnoun oranges (Citrus sinensis). Minufiya-Journal-of-AgriculturalResearch, 6: 227-239. (1983)

Anonymous: Area and production of different of different fruits in Punjab state. Directorate of Horticulture, Punjab, Chandigarh. (2019)

Bellini E, Falqui D and Musso O: Comparison between two training systems in peach 
protected culture in Sicily. Acta Hort 513: 427-33. (2000)

Boswell S B, Lewis L N, McCarty C D and Hench K W: Tree spacing of Washington Navel orange. JAm Soc Hart Sci 95 (5): 523-28. (1970).

BRAR, J.S. J.S. BAL and SOM PAL SINGH: Journal of Agrometeorology 11 (2): 135139 (2009)

Caruso T, Giovannini D, Marra F P and Sottile F: Planting density, above-ground drymatter partitioning and fruit quality in greenhouse- grown 'Flordaprince' peach (Prunus persica L. Batsch) trees trained to 'free-standing Tatura'. J Hart Sci Biotech 74 : 547-52. (1999)

Cepoiu N and Murvai M: Effect of rootstock and planting density on the growth and fruiting of some apple cultivars. LucrasiStiintifice-Institutal-Agronomic-NicolaeBalcescu-Bucuresti-seria-b-Horticulturae 31: 61-68 (1988)

Costa G, Testolin R and Sansavini S: Increasing plant density in peach: Physiological aspects, cropping and orchard management. XXII Convegno Peschicolo Cesena, Italy 28-30 September 1995, pp 130-33. (1997)

Furukowa Y and Monet R: Productivity and tree growth in high density peach orchard. Acta Hort 465: 615-19. (1998).

Hampson CR, Quamme HA and Brownlee RT: Canopy growth, yield and fruit quality of "Royal Gala" apple trees grown for eight years in five tree training systems. HortScience 37: 627-31. (2002).

Hampson CR, Quamme HA and Brownlee RT: Canopy growth, yield and fruit quality of „Royal Gala" apple trees grown for eight years in five tree training systems. HortScience 37: 627-31. (2002).

Heinicke D R: The micro-climate of fruit trees II. Foliage and light distribution pattern in apple trees. Proc Amer Soc Hort Sci 83: 1-11. (1963)

Jackson J E: Aspects of light climate wihin apple orchards. J Appl Ecol 7: 207-16. (1970)

Lal, N., Gupta, A.K., Marboh, E.S., Kumar, A.,
Nath, V: Effect of pollen grain sources on fruit set and retention in 'Rose Scented' litchi. Multilogic in Science 9 (29), 152156. 2019

Lal, N., Gupta, A.K., Marboh, E.S., Kumar, A., Nath, V: Effect of pollen grain sources on success of hybrids in 'Bedana' Litchi. International Journal of Biotic Stress Management 10(3), 241-245. 2019b

Lal, N., Gupta, A.K., Nath, V: Fruit retention in different litchi germplasm influenced by temperature. International Journal of Current Microbiology and Applied Science 6(12), 1189-1194. 2017.

Lal, N., Marboh, E.S., Gupta, A.K., Kumar, A., Dubedi Anal, A.K., Nath, V: Variation in leaf phenol content during flowering in litchi (Litchi chinensis Sonn.). Journal of Experimental Biology and Agricultural Sciences 7(6), 569-573. 2019c

Lal, N., Pandey, S.K., Nath V., Gontia, A.S., Sharma, H.L: Evaluation of litchi (Litchi chinenesis Sonn.) genotypes for fruit quality attributes. International Journal of Chemical Studies 6 (3), 2556-2560. 2018a

Lal, N., Pandey, S.K., Nath, V., Agrawal, V., Gontia, A.S., Sharma, H.L: Total phenol and flavonoids in by-product of Indian litchi: Difference among genotypes. Journal of Pharmacognosy and Phytochemistry 7(3), 2891-2894. (2018).

Lal, N., Singh, A., Gupta, A.K., Marboh, E.S., Kumar, A., Nath, V: Precocious Flowering and Dwarf NRCL-29-A New Genetic Stock of Litchi (Litchi Chinensis Sonn.). Chemical Science Review and Letters 8 (32), 206-210. (2019d)

Lal, S., et al: Tree growth, fruit quality and yield attributes as affected by tree spacing and varieties of peaches/nectarine under temperate climate. International Journal of Chemical Studies. 6(2): 308-311 (2018).

Marathe, R. A., et al: DOI: http://doi.org/10.22438/jeb/39/2/MRN511. (2017).

Nagraj, K, Diwan G, Lal N: Effect of fruit load on yield and quality of litchi (Litchi 
chinensis Sonn.). Journal of Pharmacognosy and Phytochemistry 8(6), 1929-1931 (2019).

Palmer J W, Avery D J and Wertheium S J: Effect of apple spacing and summer pruning on leaf area distribution and light interception. Sci Hort 52: 303-12. (1992).

Palmer J W: The effects of row orientation, tree height, time of year and latitude on light interception and distribution in model apple hedgerow canopies. J Hort Sci 64: 137-45. (1989)

Pandey et al: An international quarterly journal of environmental sciences Vol. VII: 397401: (2015).

Panse VG, Sukhatme PV: Statistical Methods for Agricultural Workers, Indian Council of Agricultural Research, New Delhi, 1978, 381 .

Robinson T L, Lakso A N and Carpenter S G: Canopy development, yield, and fruit quality of "Empire" and "Delicious" apple trees grown in four orchard production systems for ten years. $J A m$ Soc Hortic Sci 116: 179-87. (1991).

Rufato L, Rossi A D, Picolotto L, Parizoto E and Fachinello $\mathrm{J} \quad \mathrm{C}$ : Evaluation of vegetative and productive responses of two peach training system ( $\mathrm{Y}$ system and Central leader) in an ICM orchards. Acta Hort 636: 711-15. (2004)

Sharma J N, Chohan G S, Vij V K and Monga P $\mathrm{K}$ : Effect of spacings on growth, yield and quality of Kin now mandarin. Indian J Hart, 49: 2, 158-164 (1992).
Sharma J N, Chouhan G S, Vij V K and Monga $\mathrm{P}$ K: Pre-bearing performance of Kinnow- a mandarin at different spacings. Indian J Hart 48 (1): 8-12. (1991)

Singh $\mathrm{H}$ : Effect of planting densities and training systems on light interception, growth, productivity and nutrient composition of peach. Ph.D. Dissertation, Punjab Agricultural University, Ludhiana, India. (2001)

Singh, G., Singh, A.K. and Mishra, D: HIGH DENSITY PLANTING IN GUAVA. Acta Hortic. 735, 235-241 (2007).

Tustin S, Hurst P, Warrington I and Stanley J: Light distribution and fruit quality through multi-layered trellis apple canopies. Acta Horticulturae, 243: 20912. (1989)

Wagenmaker P Sand Callesen 0: Influence of light interception on apple yield and fruit quality related to arrangement and tree height. Acta Horticu/turae 243: 149-58. (1989)

Wagenmakers $\mathrm{P}$ S and Callesen O: Light distribution in apple orchard systems in relation to production and fruit quality. $J$ Hortic Sci 70: 935-48. (1995).

Yadav E D, Gaikwad M Rand Patil A V: The relation between tree growth, chlorophyll content and plant density of Sardar guava (Psidium guajava L.) Nat Symp Trop and Subtrop Fruit Crops, Bangalore p. 30. (1981)

\section{How to cite this article:}

Jyoti Singh, S. K. Pandey, H. K. Rai, Vishal Nath, Evening Stone Marboh and Shiv Poojan. 2020. Influence of Spacing and Planting System on Light Interception, Physiological Parameters, Yield and Quality of Litchi cv. Shahi. Int.J.Curr.Microbiol.App.Sci. 9(09): 12141226. doi: https://doi.org/10.20546/ijcmas.2020.909.149 